\title{
Commentary: Time in the therapeutic window is time well spent
}

\author{
Derrick Y. Tam, MD, and Stephen E. Fremes, MD, MSc
}

\footnotetext{
From the Schulich Heart Centre, Division of Cardiac Surgery, Department of Surgery, Sunnybrook Health Sciences Centre, University of Toronto, Toronto, Ontario, Canada.

Disclosures: Authors have nothing to disclose with regard to commercial support.

Received for publication Feb 25, 2019; accepted for publication Feb 26, 2019; available ahead of print April 4, 2019.

Address for reprints: Stephen E. Fremes, MD, MSc, Schulich Heart Centre, Sunnybrook Health Sciences Centre, 2075 Bayview Ave, Room H4 05, Toronto, Ontario, Canada M4N 3M5 (E-mail: Stephen.fremes@ sunnybrook. ca).

J Thorac Cardiovasc Surg 2020;159:84-5

$0022-5223 / \$ 36.00$

Copyright (c) 2019 by The American Association for Thoracic Surgery

https://doi.org/10.1016/j.jtcvs.2019.02.107
}

In young and middle-aged patients with unrepairable aortic or mitral valves, valve replacement with a mechanical valve prosthesis remains the treatment of choice for most patients. For the majority of patients with an aortic or mitral mechanical prosthesis, lifelong oral anticoagulant therapy with a vitamin $\mathrm{K}$ antagonist (VKA) such as warfarin is recommended. ${ }^{1}$ The use of VKA requires frequent monitoring of the international normalized ratio (INR) to ensure that the patient is within the therapeutic range. To assess the quality of VKA therapy, the time in therapeutic range (TTR) can be measured. Havers-Borgersen and colleagues ${ }^{2}$ carefully examined the association of TTR and the risk of thromboembolic events and bleeding in those with mechanical aortic or mitral valve prosthesis through linkages of administrative and clinical databases in Denmark. ${ }^{2}$

Six hundred fifty-nine patients from 1997 through 2012 were followed for 6 months after mechanical valve replacement and successive INR values were tracked. TTR was calculated from total time in the therapeutic window for the patient's valve type divided by total follow-up time. Using a cutoff of TTR $\geq 70 \%$ to define high-quality VKA therapy (consistent with European guidelines), the authors used a multivariable logistic regression model to determine baseline characteristics that predicted TTR $\geq 70 \%$ and found that only mechanical mitral valves were associated with a TTR $<70 \%$. Only $29 \%$ of patients had good-quality VKA in this 6-month analysis and the median TTR was $54.9 \%$, suggesting that for only slightly more than half the time, patients were in the therapeutic range. In a Cox-proportional cause-specific hazard model that adjusted for sex, age, valve type, and comorbidities, authors found that TTR $\geq 70 \%$ was protective against thromboembolic events (hazard ratio [HR], 0.44; 95\% confidence interval [CI], 0.22-0.85) but not bleeding (HR, 0.63 ; 95\% CI, 0.36-1.08) or all-cause mortality (HR, 0.84; 95\% CI, 0.50-1.42).

Nonetheless, these findings must be interpreted in the context of some important limitations. The time period of

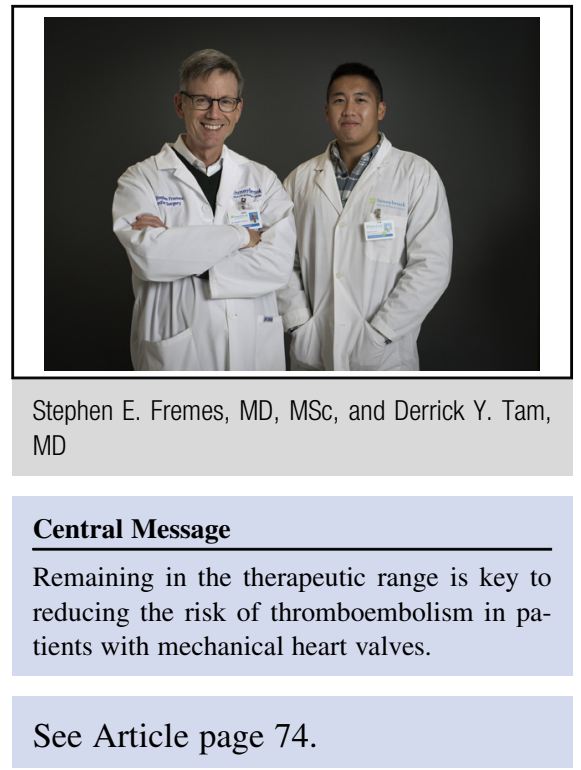

the study spanned a large range and although more than 3000 patients received a mechanical heart valve during that time, only 659 patients were included in this analysis, highlighting that the study population was a select group. Furthermore, the contemporary management of VKA may have changed substantially throughout the time period. A large retrospective study of more than 20,000 patients showed that home INR monitoring improves TTR. ${ }^{3}$ Given that INRs were obtained from administrative databases, it is very likely that home monitoring was not used in this cohort of patients. The authors chose to use a Coxproportional hazard model rather than a competing risk model in their primary analysis. Although often similar results are produced, adjusting for death as a competing risk may produce a less-biased result and yield higher precision around point estimates. The authors modeled the time to first event, yet this approach does not account for recurrent events that may be typical in this clinical scenario. Having a nonfatal event of interest likely alters clinical treatment, and thus the time to first event model used in the primary analysis is appropriate.

To their credit, the authors performed various sensitivity analyses to assess the robustness of their findings. INR variability, a measure of the degree in deviation of an INR from a previous measurement and another metric of VKA therapy quality, was not associated with the outcomes of interest. The treatment of TTR as a time-dependent covariate 
was also not a significant predictor of thromboembolism. These negative findings raise concerns regarding the robustness of the findings in a limited sample size of patients. There is a paucity of information regarding the INR values for patients outside of the therapeutic range, and such additional data would be informative and could explain the lack of statistical difference for bleeding events (eg, low INR was the reason for being out of the therapeutic range rather than a high INR).

The management of aortic valve disease in young to middle-aged patients continues to evolve at a rapid pace. The US guidelines have reduced the age threshold for a biological valve to 50 years (the median age in this study was 58 years [interquartile range, 50-64 years]); thus, there may be fewer patients undergoing mechanical aortic valve replacement to avoid the need for any VKA. ${ }^{1}$ Novel advances in valve technology include the On-X mechanical valve (CryoLife Inc, Kennesaw, Ga), which has received Food and Drug Administration and European CE mark approval for a lower range of INR (1.52.0) after 3 months of standard VKA therapy-there is evidence to suggest that lower INR therapeutic ranges reduce bleeding events without increasing the risk for thromboembolism. ${ }^{4}$
Despite these limitations, there are several important take-home messages from this study. The findings highlight the poor compliance or difficulty in staying in a therapeutic INR range and, in particular, the need for more thorough monitoring for those with a mitral mechanical prosthesis. Time in the therapeutic range appears to be a key determinant in preventing the dire complications of a mechanical prosthesis, namely thromboembolic event. In this regard, time in the therapeutic range is indeed time well spent.

\section{References}

1. Nishimura RA, Otto CM, Bonow RO, Carabello BA, Erwin JP 3rd, Fleisher LA, et al. 2017 AHA/ACC focused update of the 2014 AHA/ACC guideline for the management of patients with valvular heart disease: a report of the American College of Cardiology/American Heart Association task force on clinical practice guidelines. Circulation. 2017;70:252-89.

2. Havers-Borgersen E, Butt JH, Vinding NE, Torp-Pedersen C, Gislason G, Køber L, et al. Time in therapeutic range and risk of thromboembolism and bleeding in patients with a mechanical heart valve prosthesis. J Thorac Cardiovasc Surg. 2020;159:74-83.e4.

3. DeSantis G, Hogan-Schlientz J, Liska G, Kipp S, Sallee R, Wurster M, et al. STABLE results: warfarin home monitoring achieves excellent INR control. Am J Manag Care. 2014;20:202-9.

4. Puskas J, Gerdisch M, Nichols D, Quinn R, Anderson C, Rhenman B, et al. Reduced anticoagulation after mechanical aortic valve replacement: interim results from the prospective randomized on- $\mathrm{X}$ valve anticoagulation clinical trial randomized Food and Drug Administration investigational device exemption trial. J Thorac Cardiovasc Surg. 2014;147:1202-10. 